\title{
Simultaneous observations with a sodium lidar and an MF radar during PREASA-2 campaign
}

\author{
Hu Xiong ${ }^{1}$, Xun-jie Zhang ${ }^{1}$, Shun-sheng Gong ${ }^{1}$, and Kiyoshi Igarashi ${ }^{2}$ \\ ${ }^{1}$ Wuhan Institute of Physics and Mathematics, The Chinese Academy of Sciences, Wuhan 430071, P.R.China \\ ${ }^{2}$ Communication Research Laboratory, Ministry of Posts and Telecommunications, Tokyo 184-8795, Japan
}

(Received July 28, 1998; Revised January 14, 1999; Accepted January 14, 1999)

\begin{abstract}
Simultaneous observations with a sodium lidar in Wuhan, China $\left(30.53^{\circ} \mathrm{N}, 114.37^{\circ} \mathrm{E}\right)$ and an $\mathrm{MF}$ radar in Yamagawa, Japan $\left(31.20^{\circ} \mathrm{N}, 130.62^{\circ} \mathrm{E}\right)$ were conduced during the PREASA-2 campaign from Feb. 22 to March 15 , 1996. Some observation results are given. The gravity wave induced velocities measured by both techniques are estimated to compare the gravity wave activities between the places with same latitudes and different longitudes. We found that RMS velocities in Yamagawa were larger than those in Wuhan, which suggest different gravity wave activities between the two places.
\end{abstract}

\section{Introduction}

During Feb. 22 to March 15, 1996, the PREASA-2 (Pacific Region Equatorial Anomaly Studies in Asia) campaign was conduced to further explore the upper atmosphere by use of multi-techniques. In this campaign, at almost the same latitude, a sodium lidar in Wuhan, China, and an MF radar in Yamagawa, Japan, were operated. The distance between the two stations is about $1,563 \mathrm{~km}$. The Na layer profile, $\mathrm{Na}$ density perturbations and behavior of the gravity wave spectra by the sodium lidar have been estimated (Ai and Zhang, 1998). The MF radar winds have been compared with the MU radar winds (Igarashi et al., 1996) and analyzed to get their spectrum features (Hocke and Igarashi, 1997). While both techniques can estimate the gravity wave induced root mean squared (RMS) velocity, it is allowed a comparison during the simultaneous observations.

Comparisons between collocated lidar and $\mathrm{MF} / \mathrm{HF}$ radar can be found in several papers. Franke et al. (1990) gave a comparison of the vertical velocity measured by HF radar and that estimated by sodium lidar. Bills et al. (1991) compared the horizontal winds by MF radar and that by Doppler wind lidar. Most recently, Collins et al. (1997) presented a comparison of the gravity wave induced RMS velocity measured by MF radar and $\mathrm{Na}$ density and temperature lidar. They point out that the agreement between different techniques is good except that the RMS velocities were a little over-estimated by MF radar.

In this paper, RMS velocities simultaneously measured by the sodium lidar in Wuhan and the MF radar in Yamagawa are presented to compare the gravity wave activities in the places with the same latitudes but different longitudes.

Copy right (c) The Society of Geomagnetism and Earth, Planetary and Space Sciences (SGEPSS); The Seismological Society of Japan; The Volcanological Society of Japan; The Geodetic Society of Japan; The Japanese Society for Planetary Sciences.

\section{Experiments}

Na density profiles of the Na layer at 80-100 km level have been measured by the sodium lidar in Wuhan since the end of 1995. As the lidar was described elsewhere in details, the following is a simple description. This lidar is configured with an operating wavelength of $5890 \AA$, a pulse energy of $50 \mathrm{~mJ}$, a repeat frequency of $20 \mathrm{~Hz}$, and a telescope diameter of $40 \mathrm{~cm}$, providing the $\mathrm{Na}$ layer profile data with $4 \mathrm{~min}$ of temporal resolutions and $192 \mathrm{~m}$ of vertical resolutions.

An Na relative density profile can be calculated directly from a photoncount profile measured by the sodium lidar. After all $\mathrm{Na}$ profiles in one night are normalized by their integration sum, an averaged Na profile can be obtained. The background $\mathrm{Na}$ layer shape is usually taken as a Gaussian fit to the averaged $\mathrm{Na}$ profile over the observation period with its peak height $z_{\mathrm{m}}$ and width $\sigma_{\mathrm{m}}$. The relative $\mathrm{Na}$ density fluctuations are calculated relative to this Gaussian fit as,

$$
r(z, t)=\frac{\rho_{\mathrm{s}}^{\prime}(z, t)}{\overline{\rho_{\mathrm{s}}(z)}}=\frac{\rho_{\mathrm{s}}(z, t)-\overline{\rho_{\mathrm{s}}(z)}}{\overline{\rho_{\mathrm{s}}(z)}}
$$

where $\rho_{\mathrm{s}}(z, t)$ is one normalized Na density profile, $\overline{\rho_{\mathrm{s}}(z)}$ denotes the Gaussian fit to the mean profile, $\rho_{\mathrm{s}}^{\prime}(z, t)$ the density fluctuations.

If assumed that $\mathrm{Na}$ acts as a tracer of gravity wave motions and $\mathrm{Na}$ density perturbations are induced only by gravity waves, relative atmospheric density perturbations can be derived under a linear approximation as (Gardner et al., 1989),

$$
\left[\frac{\rho_{\mathrm{a}}^{\prime}(z, t)}{\overline{\rho_{\mathrm{a}}(z)}}\right]^{2}=\Gamma^{-2}(z) r^{2}(z, t)
$$

where

$$
\Gamma(z)=-\frac{1}{\gamma-1}\left[1-\frac{\gamma H}{\sigma_{\mathrm{m}}^{2}}\left(z-z_{\mathrm{m}}\right)\right] .
$$

$\rho_{\mathrm{a}}^{\prime}(z, t)$ denote atmospheric density perturbations, $\gamma(=1.4)$ the adiabatic rate, and $H(\approx 6 \mathrm{~km})$ the density scale height. 
By using of gravity wave dispersion relationships, a mean square horizontal velocity variance is derived as,

$$
\overline{u^{\prime}(z)^{2}}=\left(\frac{g}{N}\right)^{2} \overline{\left[\frac{\rho_{\mathrm{a}}^{\prime}(z, t)}{\overline{\rho_{\mathrm{a}}(z)}}\right]^{2}}
$$

where $g\left(\approx 9.53 \mathrm{~m} / \mathrm{s}^{2}\right)$ is the gravity acceleration, the B-V frequency, $N^{2}$, is taken to be $4.1 \times 10^{-4} \mathrm{~s}^{-2}$ for simplicity. It is worth noting that Eq. (2) is no longer valid at a height where $\Gamma$ becomes zero as $z$ approaches to $z_{0}=z_{\mathrm{m}}+\sigma_{\mathrm{m}}^{2} /(\gamma H)$, since $\mathrm{Na}$ density perturbations response nonlinearly to gravity waves. In this paper, RMS velocities near this height are not obtained due to this limitation.

Mesospheric winds have been measured by the MF radar in Yamagawa since Sept., 1994. This MF radar is configured with three receivers and one transmitter, and the FCA method is adopted to calculate the winds. Its operating frequency is $1.955 \mathrm{MHz}$ with a pulse peak power of $50 \mathrm{Kw}$, and it provides winds data from $60 \mathrm{~km}$ to $98 \mathrm{~km}$ level with resolutions of $2 \mathrm{~km}$ in vertical and $2 \mathrm{~min}$ in time.

The mesospheric winds measured by MF radar usually have contributions by gravity waves, tides and planetary waves. For one day's data, the following fitting function is used to get the tides and a linear trend,

$$
\begin{aligned}
y(t)= & A_{0}+b \times t+A_{8} \times \cos \left(\omega_{8} t+\varphi_{1}\right) \\
& +A_{12} \times \cos \left(\omega_{12} t+\varphi_{2}\right) \\
& +A_{24} \times \cos \left(\omega_{24} t+\varphi_{3}\right)
\end{aligned}
$$

where $A_{0}$ is the mean, $b$ the trend, and $A_{8}$ is the amplitude of $8 \mathrm{~h}$ tidal wave, $A_{12}$ the amplitude of $12 \mathrm{~h}$ tidal wave, $A_{24}$ the amplitude of $24 \mathrm{~h}$ tidal wave, and $\omega_{8}, \omega_{12}, \omega_{24}$ and $\varphi_{1}, \varphi_{2}, \varphi_{3}$ are their angular frequencies and phases respectively. After gravity wave components are calculated by subtracting the tides and trend from the original winds data, RMS velocities can be obtained.

\section{Observation Results and Conclusion}

The observations on the night of March 2, 1996 were selected for comparison. In this night, the lidar obtained 36 profiles starting at 12:15UT with a time resolution of $4 \mathrm{~min}$. The MF radar operated routinely during this period.

Figure 1 shows the averaged profile of the $\mathrm{Na}$ layer and its Gaussian fitted function. The vertical axis is normalized and has no dimension. It can be inferred from Fig. 1 that the peak height $z_{\mathrm{m}}$ of $\mathrm{Na}$ layer is $92.840 \pm 0.066 \mathrm{~km}$, and the width $\sigma_{\mathrm{m}}$ of the peak is $4.285 \pm 0.066 \mathrm{~km}$.

Figure 2 gives RMS velocity profiles measured by the lidar and the MF radar respectively. The RMS velocities measured by lidar were averaged every $2 \mathrm{~km}$. A gape between 93 $\mathrm{km}$ and $96 \mathrm{~km}$ is due to the invalid of Eq. (2), in which $z_{0} \approx 95.026 \pm 0.133 \mathrm{~km}$. Their horizontal bars denote $95 \%$ confidence. For comparison, The RMS velocities measured by MF radar averaged in almost the same time range of 11UT$14 \mathrm{UT}$ as that by lidar were depicted, and a profile of one-day averaged RMS velocities by MF radar was also depicted with their $90 \%$ confidence bars in the same figure. Gaps in MF radar data may bring error in estimations of the RMS velocity and this error is not evaluated in this paper.

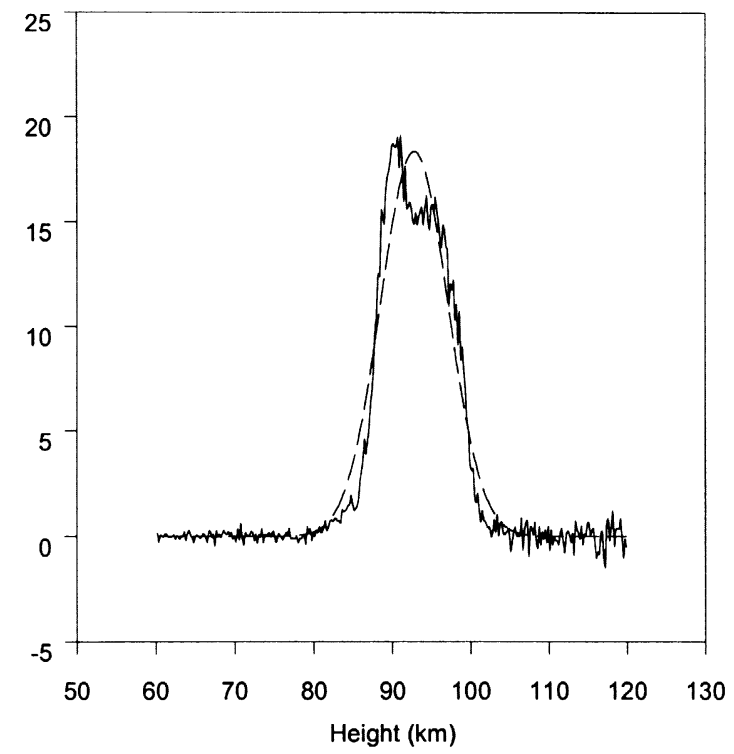

Fig. 1. Normalized mean Na density profile (solid line) and its Gaussian fitted function (dashed line).

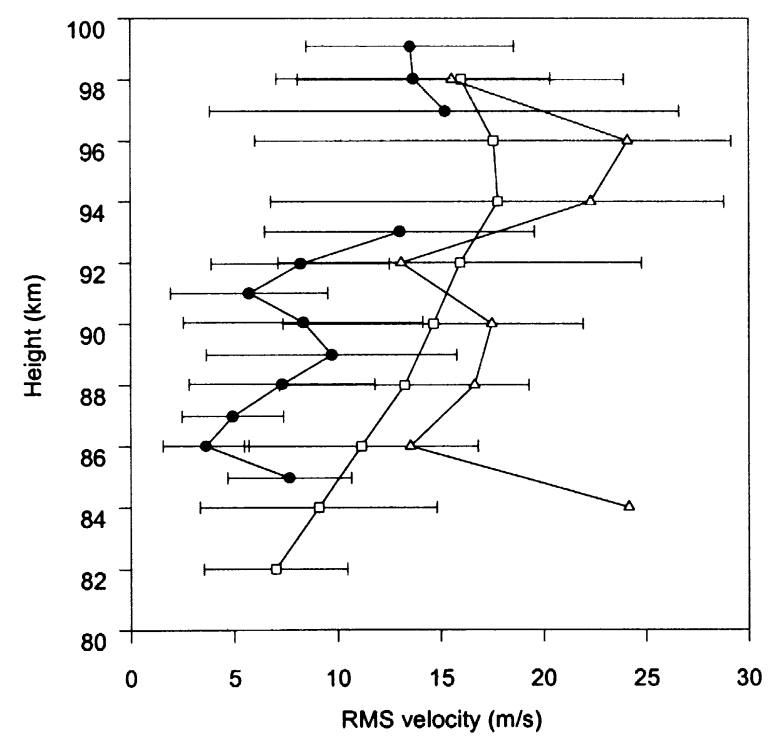

Fig. 2. Comparison of RMS velocity measurements between an $\mathrm{Na}$ lidar and an MF radar. Solid circles with $95 \%$ confidence bars denote the lidar measurements, squares with $90 \%$ confidence bars denote the one-day average measurements by MF radar, and triangles denote the 11-14UT average measurements by MF radar.

It is clear from Fig. 2 that RMS velocities by MF radar in the same time range are greater than those by lidar. Because the atmospheric density and temperature profiles are not available in Wuhan, the $N^{2}$ and scale height $H$ are taken as constant values for all heights in the lidar's RMS velocities estimations, which may brought some errors to calculations as well as the applied linear approximation. The accuracy of the lidar RMS velocity estimations also depends on the Gaussian fit methods we used and alternative fit methods will be 
adopted to promote the accuracy in our future works. Under considering these aspects, it can be inferred that differences in RMS velocities might show different gravity wave activities between the two places. Actually, the two places have a great distance more than $1,500 \mathrm{~km}$ between. At so long a distance, their gravity wave sources might be different and it is not a question that their gravity wave activities could be different as observations given above.

One interesting point is that their vertical profiles show similar wavelike structures, which may imply that a kind of inertia gravity waves with an about $5 \mathrm{~km}$ vertical wavelength and a period larger than the observation period prevailed above Wuhan and Yamagawa respectively at that time.

The amplitudes of one day averaged RMS velocities by MF radar increase with increasing height below $95 \mathrm{~km}$, which suggests saturation of gravity waves, i.e., that amplitudes of gravity wave activities increase with height and become saturated at $95 \mathrm{~km}$ altitude.

The above comparison of RMS velocities measured by the lidar and MF radar shows that the gravity wave activities are different between the two places with different longitudes in the night of March 2, 1996. Further comparisons of future simultaneous observations are needed to support these contentions.

\section{References}

Ai, Y. and X. Zhang, Characteristics of atmospheric density spectra in the mesopause region at Wuhan, China during March 1996, Earth Planets Space, 50, 383-386, 1998.

Bills, R. E., C. S. Gardner, and C. Y. She, Narrowband lidar technique for Na temperature and Doppler wind observations of the upper atmosphere, Opt. Eng., 30, 13-21, 1991.

Collins, R., D. Thorsen, and S. J. Franke, Comparative MF radar and Na lidar measurements of fluctuating winds in the mesopause region, J. Geophys. Res., 102, D14, 16,583-16,591, 1997.

Franke, S. J., T. Beatty, D. Thorsen, C. H. Liu, and C. S. Gardner, Simultaneous Na lidar and HF radar observations of vertical velocities in the mesosphere above Urbana.Illinois, Geophys. Res. Lett., 17, 1, 69-72, 1990.

Gardner, C. S., D. C. Senft, T. J. Beatty, R. E. Bills, and C. A. Hostetler, Rayleigh and sodium lidar techniques for measuring middle atmosphere density, temperature and wind perturbations and their spectra, WITS Handbook, 2, 148-187, 1989.

Hocke, K. and K. Igarashi, Variability and anisotropy of mesospheric wind spectra, Geophys. Res. Lett., 24, 2725-2728, 1997.

Igarashi, K., I. Nishimuta, Y. Murayama, T. Tsuda, T. Nakamura, and M. Tsutsumi, Comparison of wind measurements between Yamagawa MF radar and the MU radar, Geophys. Res. Lett., 23, 3341-3344, 1996.

H. Xiong (e-mail: hx@nmr.whcnc.ac.cn), X. Zhang, S. Gong, and K. Igarashi 